\title{
Barrier responses of human bronchial epithelial cells to grass pollen exposure
}

\author{
Cornelia Blume1, Emily J. Swindle ${ }^{1}$, Patrick Dennison ${ }^{1,2}$, Nivenka P. Jayasekera1, \\ Sarah Dudley ${ }^{3}$, Phillip Monk ${ }^{3}$, Heidrun Behrendt ${ }^{4}$, Carsten B. Schmidt-Weber ${ }^{4}$, \\ Stephen T. Holgate ${ }^{1,2}$, Peter H. Howarth², Claudia Traidl-Hoffmann ${ }^{4}$ and \\ Donna E. Davies ${ }^{1,2}$
}

\begin{abstract}
Affiliations: 'Brooke Laboratory, Clinical and Experimental Sciences, Faculty of Medicine, University Hospital Southampton, Southampton, ${ }^{2}$ National Institute for Health Research Respiratory Biomedical Research Unit, University Hospital Southampton, Southampton, and ${ }^{3}$ Synairgen Research Ltd, University Hospital Southampton, Southampton, UK. ${ }^{4}$ Centre for Allergy and Environment (ZAUM), Technische Universität Munich/Helmholtz Centre, Munich, Germany.
\end{abstract}

Correspondence: D.E. Davies, Clinical and Experimental Sciences, Sir Henry Wellcome Laboratories, Mailpoint 810, Level F, South Block, University Hospital Southampton, Southampton S016 6YD, UK. E-mail: donnaddasoton.ac.uk

ABSTRACT The airway epithelium forms a physical, chemical and immunological barrier against inhaled environmental substances. In asthma, these barrier properties are thought to be abnormal. In this study, we analysed the effect of grass pollen on the physical and immunological barrier properties of differentiated human primary bronchial epithelial cells.

Following exposure to Timothy grass (Phleum pratense) pollen extract, the integrity of the physical barrier was not impaired as monitored by measuring the transepithelial resistance and immunofluorescence staining of tight junction proteins. In contrast, pollen exposure affected the immunological barrier properties by modulating vectorial mediator release. CXC chemokine ligand (CXCL)8/interleukin (IL)- 8 showed the greatest increase in response to pollen exposure with preferential release to the apical compartment. Inhibition of the extracellular signal-regulated kinase $1 / 2$ and p38 mitogen-activated protein kinase pathways selectively blocked apical CXCL8/IL-8 release via a post-transcriptional mechanism. Apical release of CC chemokine ligand (CCL)20/macrophage inflammatory protein-3 $\alpha$, CCL22/monocyte-derived chemokine and tumour necrosis factor- $\alpha$ was significantly increased only in severe asthma cultures, while CCL11/eotaxin-1 and CXCL10/interferon- $\gamma$-induced protein-10 were reduced in nonasthmatic cultures.

The bronchial epithelial barrier modulates polarised release of mediators in response to pollen without direct effects on its physical barrier properties. The differential response of cells from normal and asthmatic donors suggests the potential for the bronchial epithelium to promote immune dysfunction in asthma.

@ERSpublications

Bronchial epithelial barrier response to pollen exposure: does bronchial epithelium promote immune dysfunction in asthma? http://ow.ly/kFDjR

This article has supplementary material available from www.erj.ersjournals.com

Received: May 112012 | Accepted after revision: Oct 152012 | First published online: Nov 082012

Support statement: Funded by the Medical Research Council (UK; grant number G0800649), Synairgen Research Ltd, Bayerische Forschungsstiftung (Germany; PDOC-65-09), CK-CARE of the Kuehne Foundation (Switzerland) and SFB/ TR22 (Germany). Stephen T. Holgate is a Medical Research Council Clinical Professor.

Conflict of interest: None declared.

Copyright @ERS 2013 


\section{Introduction}

Asthma is a chronic inflammatory disorder of the conducting airways that is characterised by bronchial hyper-reactivity in response to innocuous stimuli causing variable airflow obstruction. It has been hypothesised that the airway epithelium plays a key role in the development of asthma [1], since it is the first site of contact with environmental substances and expresses several of the recently identified asthma susceptibility genes. The barrier functions of the bronchial epithelium can be separated into three types: chemical, physical and immunological [2]. The chemical barrier is provided by mucous secretions of bronchial epithelial cells and contains, among others, antimicrobial molecules, antioxidants and protease inhibitors that contribute to the innate defence [2]. The physical barrier is mainly formed by intercellular junctions, particularly tight junctions, adherens junctions and desmosomes. Tight junctions are located at the most apical side of the cell layer, forming the closest site of contact between neighbouring cells and regulating the macromolecular and ionic permeability and polarity of the epithelial barrier [3]. Tight junction complexes are formed by transmembrane spanning proteins, including claudins, occludin and junctional adhesion molecule, which link neighbouring cells. On the intracellular side, these transmembrane proteins are connected to the actin cytoskeleton via protein complexes including zonula occludens (ZO) proteins. In addition, bronchial epithelial cells are able to influence the immunological barrier through their ability to release immunostimulatory and immunomodulatory mediators such as cytokines, chemokines and growth factors. In asthma, there is evidence that the epithelial barrier properties are impaired, which may be caused by intrinsic factors, e.g. genetic polymorphisms [4], or by environmental factors such as respiratory viruses, cigarette smoke, pollution and allergens [5-8].

While asthma is a chronic condition, it is a disease that is punctuated by acute exacerbations that are severe enough to require a visit to the emergency department and which can sometimes be fatal [9]. Amongst the triggers of asthma exacerbations are respiratory viruses, air pollutants, indoor and outdoor allergens, drugs and emotional stress. While some aeroallergens, especially pollen, are more usually recognised as triggers of allergic rhinitis in susceptible individuals, studies in Canada and Australia have highlighted the increased risks of acute asthma exacerbations in the pollen season [10-12]. Besides their modulatory effect on immune cells [13], components of pollen also have the potential to affect the epithelial barrier. Using epithelial cell lines, such as Calu-3, it has been suggested that proteases released by pollen interfere with epithelial barrier functions $[14,15]$. However, these cell line-based models do not recapitulate the in vivo epithelium: they are undifferentiated, lacking cilia, goblet cells and mucus secretions that protect the cell surface from components of the inhaled environment [16]. Since differentiated primary bronchial epithelial cells (PBECs) reflect better the in vivo situation, we compared the impact of grass pollen extract on bronchial epithelial barrier functions using in vitro differentiated cells derived from severe asthmatic and healthy volunteers. We show that while the integrity of the physical barrier is not affected, the immunological barrier becomes activated after exposure to pollen-derived substances. We observed vectorial release of mediators mainly to the apical compartment after pollen exposure. Irrespective of disease, the highest increase after pollen exposure was observed for CXC chemokine ligand (CXCL)8/ interleukin (IL)-8; however, apical release of CC chemokine ligand (CCL)20/macrophage inflammatory protein (MIP)-3 $\alpha$, tumour necrosis factor (TNF)- $\alpha$ and CCL22/monocyte-derived chemokine (MDC) was only observed using cultures from severe asthmatic donors. To our knowledge this is the first report analysing the effect of pollen-derived substances on the barrier functions of differentiated PBECs.

\section{Methods}

\section{Subjects and primary cell culture}

Human PBECs were obtained by epithelial brushing using fibreoptic bronchoscopy from subjects selected from a volunteer database. All procedures were approved by the Southampton and South West Hampshire Research Ethics Committee and were undertaken following informed consent. PBECs were cultured in bronchial epithelial growth medium (Lonza, Basel, Switzerland) and differentiated at the air-liquid interface (ALI) as previously described [8]. Details of donors' phenotypes and primary cell culture are available in the online supplementary material (online supplementary tables E1 and E2).

\section{Stimulation of ALI cultures}

Timothy grass (Phleum pratense) pollen extracts were prepared as described in the online supplementary material. Fully differentiated epithelial cultures were starved for $24 \mathrm{~h}$ and exposed apically to pollen extract after an apical wash with Hanks' balanced salt solution (HBSS) to remove mucus secretions. Transepithelial resistance (TER) was monitored before stimulation and over time. At the end of the incubation, apical and basolateral secretions were harvested and cells processed for immunofluorescence staining, Western blot analysis or mRNA isolation, as described in the online supplementary material. Involvement of mitogen-activated protein kinase (MAPK) 
signalling pathways in the regulation of barrier functions was analysed using specific pharmacological inhibitors, as described in the online supplementary material.

\section{Detection of mediator release}

The concentrations of 11 different mediators in the apical and basolateral secretions were analysed. CXCL8/ IL-8 was measured using the Human CXCL8/IL-8 DuoSet ELISA kit (R\&D Systems, Abingdon, UK). Levels of CCL2/monocyte chemoattractant protein (MCP)-1, CCL5/RANTES, CCL11/eotaxin, CCL17/thymusand activation-regulated chemokine (TARC), CCL20/MIP-3 $\alpha$, CCL22/MDC, CXCL10/interferon- $\gamma$ induced protein (IP)-10, CXCL11/interferon-inducible T-cell- $\alpha$ chemoatrractant, granulocyte-macrophage colony stimulating factor (GM-CSF) and TNF- $\alpha$ were analysed using a 10-Plex kit from Meso Scale Discovery (Gaithersburg, MD, USA) according to the manufacturer's instructions.

\section{Statistical analysis}

Statistical evaluation was performed using SPSS (SPSS Inc., Chicago, IL, USA). The nonparametric MannWhitney U-test was used for independent samples and the Wilcoxon test for related samples. Differences were regarded as significant when $\mathrm{p} \leqslant 0.05$.

\section{Results}

Characterisation of ALI cultures derived from nonasthmatic and severe asthmatic individuals After differentiation at the ALI for 21 days, epithelial cultures from nonasthmatic and severe asthmatic donors were characterised by immunostaining and the barrier properties monitored by measuring the TER. As shown in figure 1a, the proportion of ciliated (i.e. $\beta$-tubulin positive) cells in ALI cultures from severe asthmatic donors was not significantly different from that found in cultures from nonasthmatic donors. In contrast, ALI cultures of severe asthmatic donors had a significantly higher percentage of mucus-producing goblet cells (i.e. MUC5AC-positive cells). Analysis of the physical barrier properties of these cultures showed
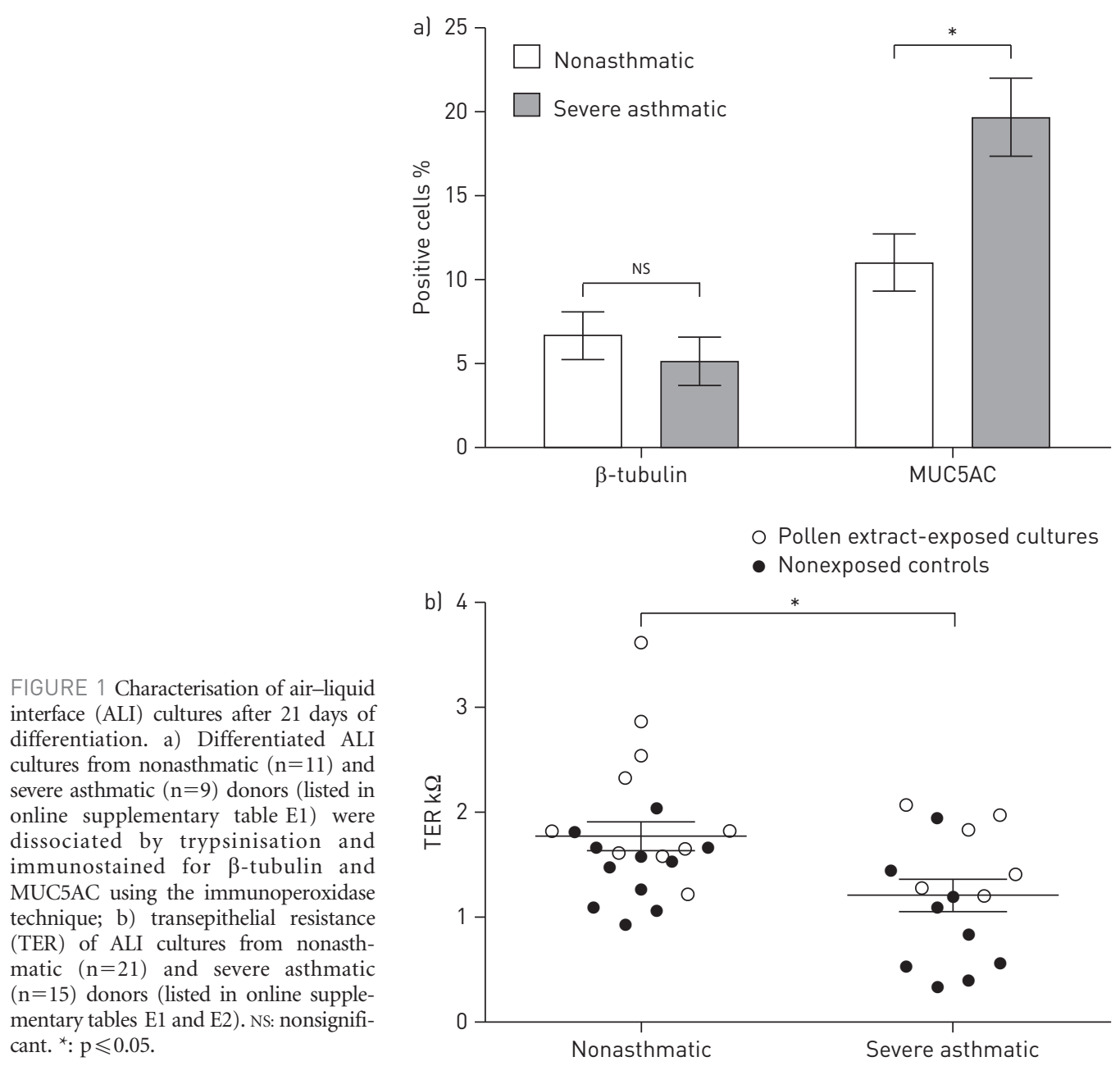
that those from severe asthmatic donors had a significantly lower TER compared with those from nonasthmatic controls (fig. 1b). These findings are consistent with those reported previously $[8,17]$.

\section{Physical barrier functions of differentiated epithelial cultures are not impaired after exposure to grass pollen extract}

The impact of grass pollen-derived substances on the physical barrier of ALI cultures was monitored by measuring TER. To analyse the change in TER over time, the apical surface of ALI cultures from nonasthmatic or severe asthmatic donors were exposed to pollen extract (equivalent to an exposure of $1 \mathrm{mg}$ of pollen grains) and the TER measured immediately after addition of the pollen $(t=0)$ and then every hour for $6 \mathrm{~h}$ without removal of the pollen extract. Under these conditions, there was an increase in TER relative to $t=0$, irrespective of disease status, reaching a plateau after $2 \mathrm{~h}$ (fig. 2a). However, this increase in TER was lost after the apical surface was washed, suggesting that the pollen extract caused a change in the conductivity of the apical medium by affecting either epithelial ion transport and/or secretion of protein polyelectrolytes. Since we observed no decrease in TER in these short-term exposures (even after replacement of the apical secretions), the cultures were challenged with a range of doses of pollen for $24 \mathrm{~h}$ and then the apical surface washed and replaced with fresh HBSS prior to TER measurement. Under these conditions, the TER did not significantly change after exposure to pollen and no significant differences were observed between nonasthmatic and severe asthmatic cultures (fig. 2b). Even repeated exposure to pollen extract (equivalent to $1 \mathrm{mg}$ of pollen grains per day) over 4 days caused no change in TER compared to the untreated control (fig. 2c).

Besides measuring TER, the integrity of the tight junctions formed by ALI cultures was analysed by immunofluorescence staining for ZO-1 and occludin. After 24 h exposure, ZO-1 (fig. 3) and occludin (data not shown) were localised at the apical side of the cell layer at cell-cell contacts and showed little difference from the untreated control. In addition, the organisation and distribution of actin filaments was not changed after treatment with pollen extract (fig. 3); in all cases the cell layer showed a strong actin filament ring in close proximity to $\mathrm{ZO}-1$, suggesting no obvious alterations in the tight junction assembly. Consistent with the lack of any apparent effect of pollen extract on tight junctions, analysis of protease activity in the pollen extract revealed only low levels of activity (equivalent to $0.46 \mu \mathrm{g} \cdot \mathrm{mL}^{-1}$ trypsin), which was just above the lower limit of detection of the assay $\left(0.10 \mu \mathrm{g} \cdot \mathrm{mL}^{-1}\right.$ trypsin) (online supplementary fig. E1), suggesting that pollen extract was not a potent source of protease activity.

Immunological barrier functions of differentiated PBECS are altered after exposure to pollen extract Since epithelial cells are able to change the properties of the immunological barrier by releasing a variety of mediators, we analysed the release of 11 different inflammation-related mediators from ALI cultures derived from nonasthmatic and severe asthmatic donors. At baseline, the concentrations of mediators released by ALI cultures were in general higher in the apical compartment compared to the basolateral compartment (fig. 4). While the rank order of cytokine secretion was similar for the apical and basolateral compartments,
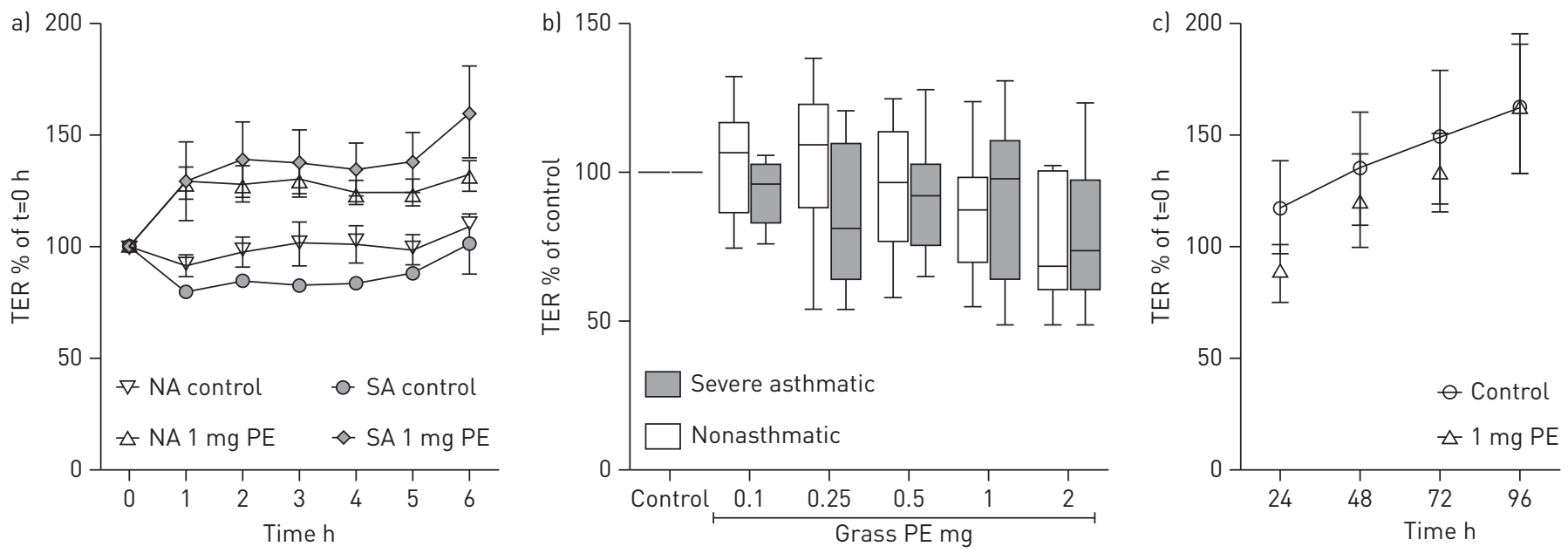

FIGURE 2 Effect of grass pollen on the transepithelial resistance (TER) of differentiated primary bronchial epithelial cells. a) TER of air-liquid interface (ALI) cultures from nonasthmatic (NA) $(n=4)$ and severe asthmatic $(\mathrm{SA})(\mathrm{n}=3)$ subjects over $6 \mathrm{~h}$ continuous exposure to $1 \mathrm{mg}$ pollen equivalent (PE). TER was normalised against the TER at $\mathrm{t}=0 \mathrm{~h}$. b) TER of ALI cultures from nonasthmatic $(\mathrm{n}=10)$ and severe asthmatic $(\mathrm{n}=7)$ subjects after $24 \mathrm{~h}$ exposure to a range of concentrations of pollen extract; TER was measured after washing the apical surface and was normalised against the TER at $0 \mathrm{~h}$ and the control at $24 \mathrm{~h}$. c) TER after apical wash of nonasthmatic ALI cultures $(n=6)$ exposed to $1 \mathrm{mg}$ pollen equivalent over 4 days. TER was normalised against the TER at 0 h. 
Control
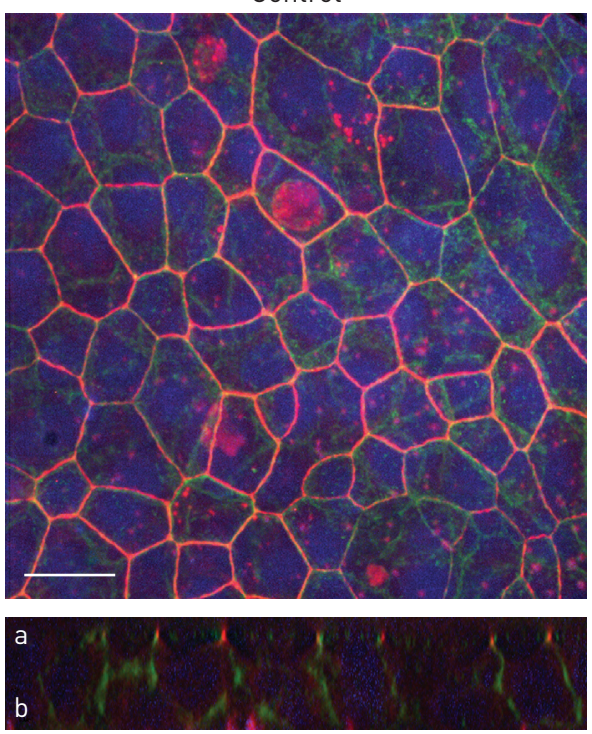

$1 \mathrm{mg} P E$
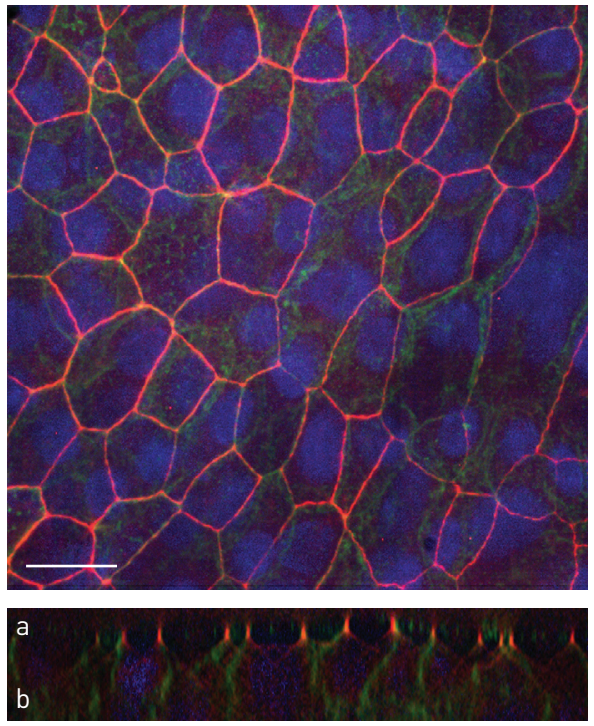

$2 \mathrm{mg} P \mathrm{P}$
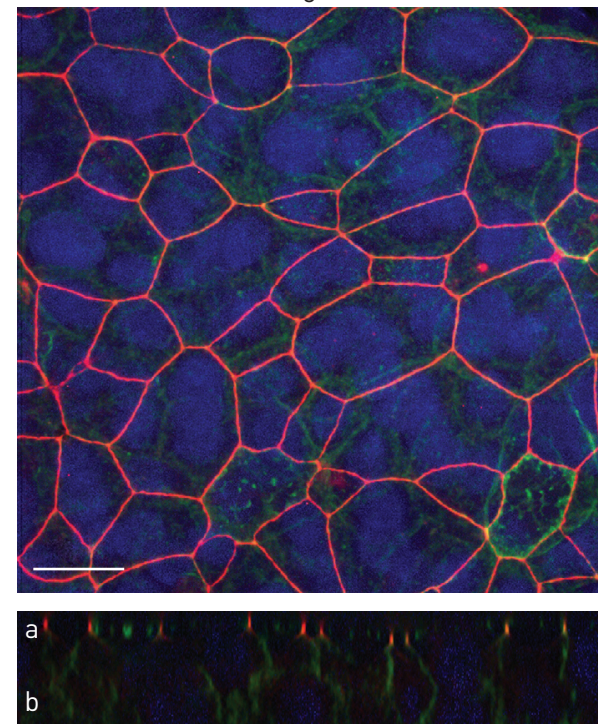

FIGURE 3 Barrier integrity of differentiated primary bronchial epithelial cells after exposure to grass pollen. Air-liquid interface cultures were exposed for $24 \mathrm{~h}$ to grass pollen (equivalent to $1 \mathrm{mg}$ pollen grains) and stained for the tight junction protein zonula occludens (ZO)-1 and actin filaments by immunofluorescence. ZO-1 staining is shown in red, actin filaments in green and nuclei are stained blue. Images of a severe asthmatic donor are shown and are representative of three. PE: pollen equivalent; a: apical; b: basal. Scale bars $=20 \mu \mathrm{m}$.
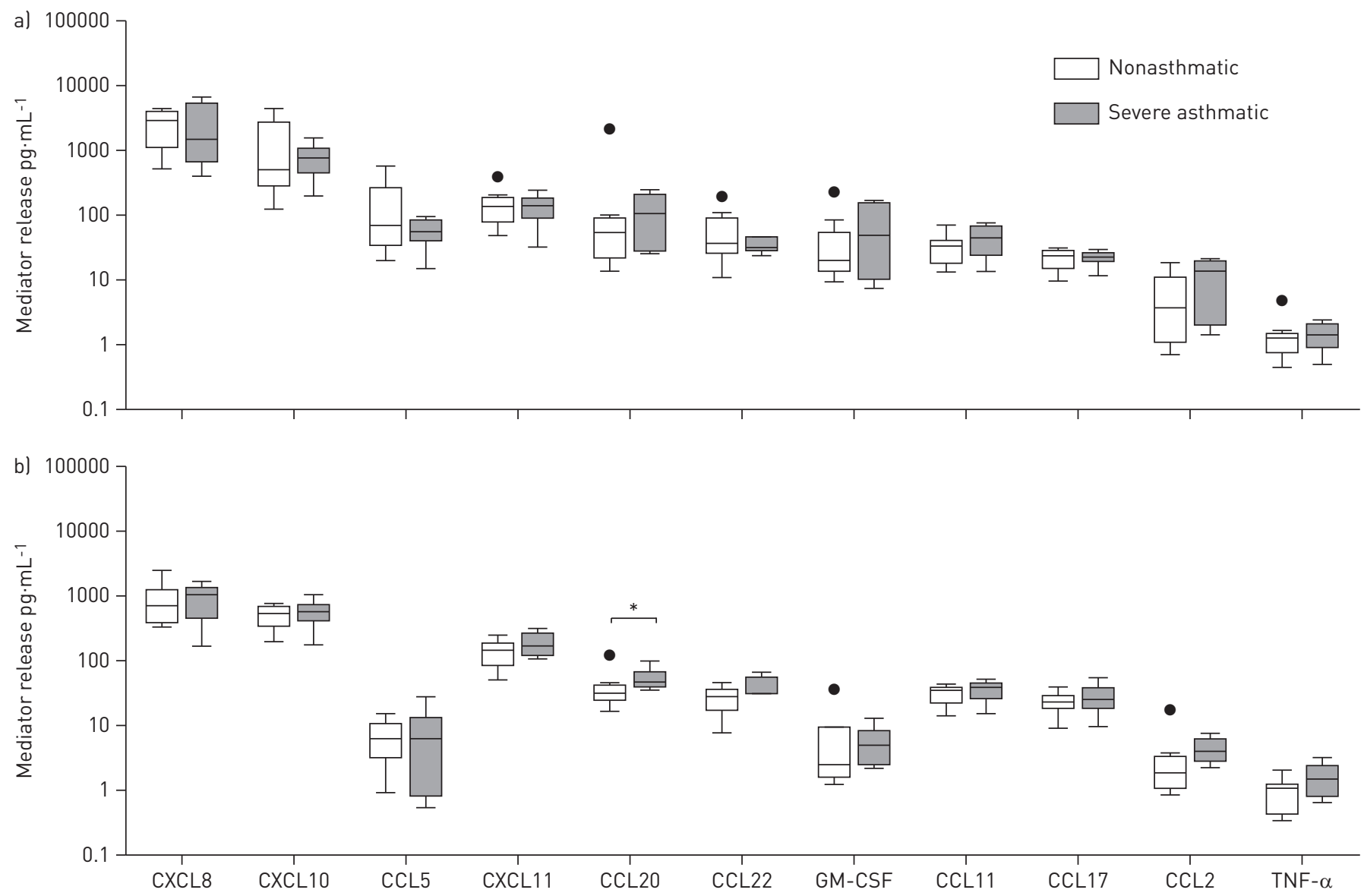

FIGURE 4 Release of mediators by differentiated bronchial epithelial cells derived from healthy and severe asthmatic subjects at baseline level. The a) apical and b) basolateral release of mediators from unstimulated air-liquid interface cultures from nonasthmatic $(n=10)$ and severe asthmatic $(n=6)$ donors (listed in online supplementary table E2) after $24 \mathrm{~h}$ were analysed by ELISA and multiplex analysis. CXCL: CXC chemokine ligand; CCL: CC chemokine ligand; GM-CSF: granulocyte-macrophage colony stimulating factor; TNF: tumour necrosis factor. ${ }^{*}: \mathrm{p} \leqslant 0.05$. 

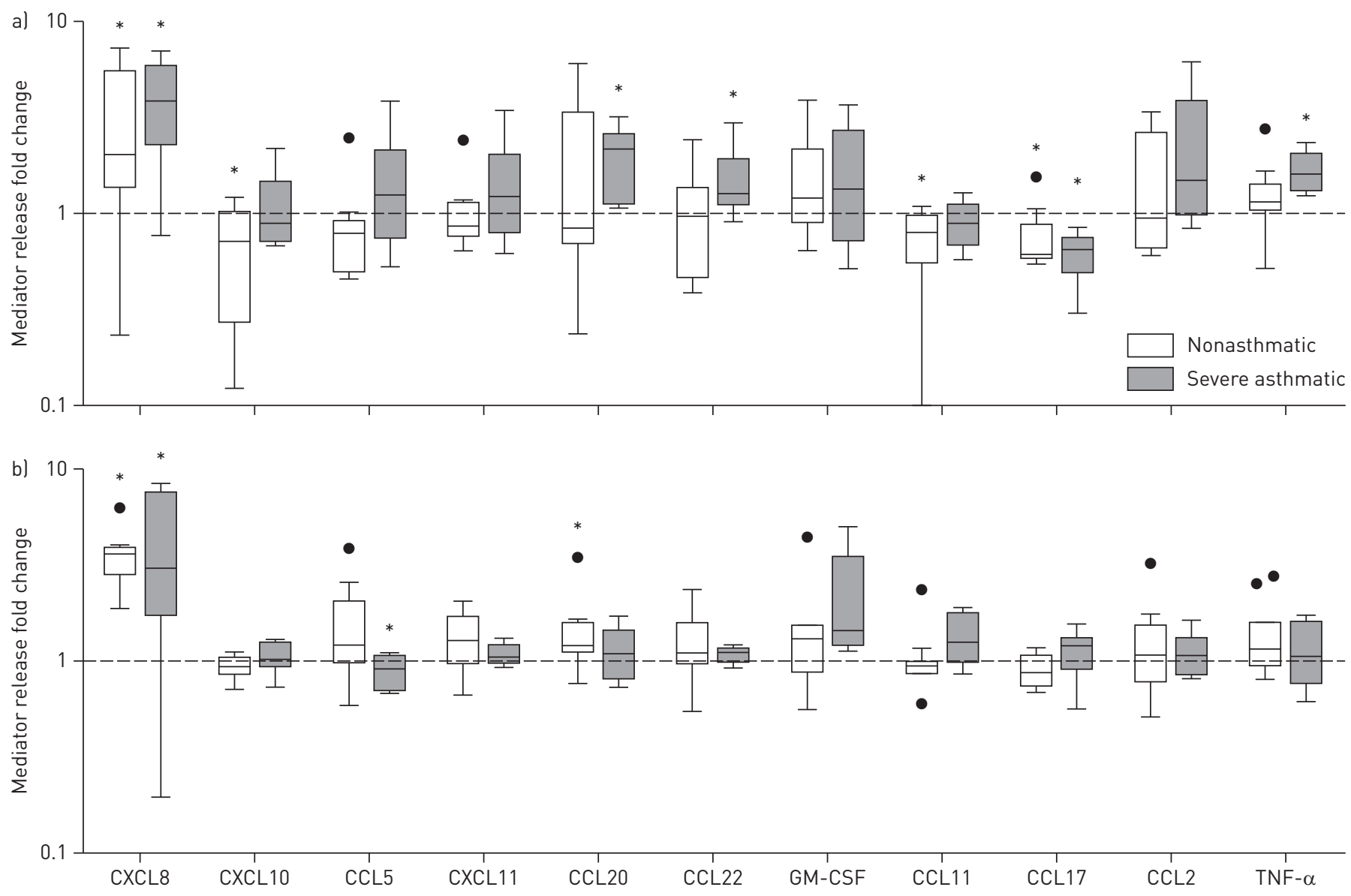

FIGURE 5 Profile of released mediators by differentiated primary bronchial epithelial cells is changed after exposure to pollen extract. a) Apical and b) basolateral release of mediators from air-liquid interface cultures derived from nonasthmatic $(\mathrm{n}=10)$ and severe asthmatic $(\mathrm{n}=6)$ subjects (listed in online supplementary table E2) was analysed after $24 \mathrm{~h}$ apical exposure to an equivalent of $1 \mathrm{mg}$ grass pollen. Mediator release was normalised to the respective control. CXCL: CXC chemokine ligand; CCL: CC chemokine ligand; GM-CSF: granulocyte-macrophage colony stimulating factor; TNF: tumour necrosis factor *: $\leqslant \leqslant 0.05$ compared to control.

there were two notable exceptions: CCL5/RANTES and GM-CSF were both significantly lower in the basolateral compartment compared with the apical compartment. In the steady state we only observed a significant difference in the basolateral release of CCL20/MIP-3 $\alpha$ between ALI cultures of nonasthmatic and severe asthmatic donors (online supplementary fig. E2a). The basolateral release of CCL2/MCP-1 and TNF- $\alpha$ tended to be increased in ALI cultures of severe asthmatic donors; however, this effect was not statistically significant (online supplementary fig. E2b and c). All other mediators showed no differences between ALI cultures derived from nonasthmatic and severe asthmatic donors.

After exposure of ALI cultures to pollen extract, the release of mediators to the apical and basolateral compartment was changed (fig. 5), especially in the apical compartment. For example, there was a 2-3-fold increase in apical CXCL8/IL-8 release, although there was no significant disease-related difference. The apical release of CCL20/MIP-3 $\alpha$, CCL22/MDC and TNF- $\alpha$ showed a 1.5-2-fold significant increase following exposure to pollen extract only in ALI cultures from severe asthmatic donors (online supplementary fig. E3) but there was no significant difference between the groups. While release of most mediators tended to increase, release of CCL17/TARC to the apical compartment was significantly reduced in ALI cultures regardless of subject group, whereas release of CXCL10/IP-10 and CCL11/eotaxin to the apical compartment was significantly decreased only in ALI cultures from nonasthmatic subjects (online supplementary fig. E4).

In contrast to the release of mediators into the apical compartment, only a few mediators showed an increase in release into the basolateral compartment after pollen exposure (fig. 5b). The largest increase was for CXCL8/IL-8, which showed a 3-4-fold induction. The other mediator showing an increase was GM-CSF, but this was only observed using ALI cultures from patients with severe asthma. We further observed a significant reduction in CCL5/RANTES release from ALI cultures of severe asthmatic donors after pollen exposure, 
FIGURE 6 Pollen dose dependently induces CXC chemokine ligand (CXCL)8/interleukin (IL)-8 release in differentiated bronchial epithelial cells from nonasthmatic and severe asthmatic subjects. Air-liquid interface cultures derived from nonasthmatic $(n=10)$ and severe asthmatic $(n=8)$ subjects were apically exposed to increasing concentrations of grass pollen extract. a) Apical and b) basolateral CXCL8/IL-8 release was analysed after $24 \mathrm{~h}$ by ELISA. *: $\mathrm{p}<0.05$.
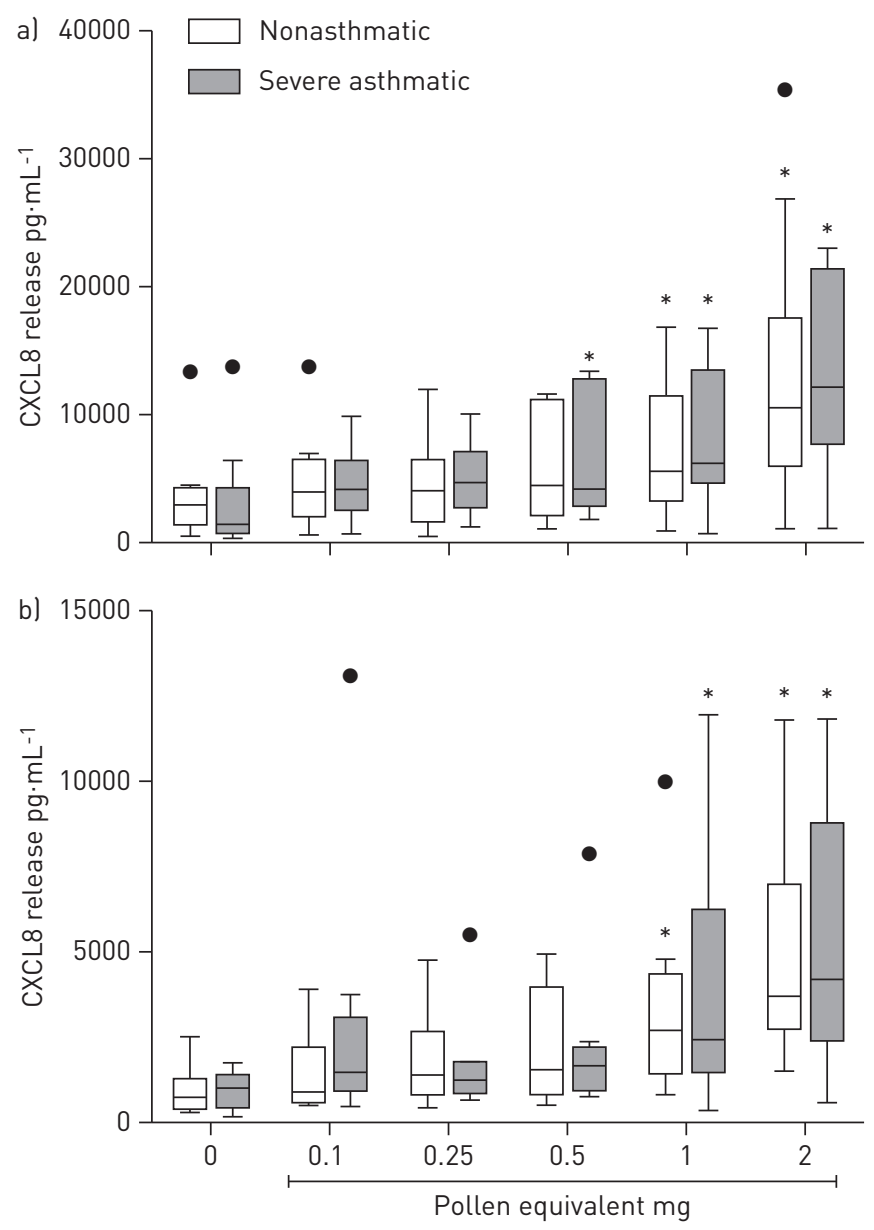

although the fold change was small compared to the control. A minor but statistical significant increase was also observed for basolateral CCL20/MIP-3 $\alpha$ release only in ALI cultures of nonasthmatic donors.

\section{Characterisation of CXCL8/IL-8 release by differentiated PBECs after exposure to pollen extract}

Since the release of CXCL8/IL- 8 by ALI cultures showed the highest increase after pollen exposure, we further characterised pollen extract-induced CXCL8/IL-8 release. As shown in figure 6, pollen extract dose dependently induced apical and basolateral release of CXCL8/IL-8 by ALI cultures derived from nonasthmatic as well as severe asthmatic donors. Cultures from both nonasthmatic and severe asthmatic subjects showed a significant increase in the apical and basolateral CXCL8/IL-8 release after exposure to pollen extract of an equivalent to $1 \mathrm{mg}$ pollen grains.

In order to analyse the signalling pathways involved in grass pollen-induced CXCL8/IL- 8 release by ALI cultures, we used specific pharmacological inhibitors of MAPK signalling pathways (fig. 7a). U0126, a specific inhibitor of the extracellular signal-regulated kinase (ERK) $1 / 2$ MAPK signalling pathway reduced pollen extract-induced apical CXCL8/IL-8 release from ALI cultures, while SB203580, a specific inhibitor of the p38 MAPK signalling pathway, completely blocked release. Inhibition of the c-jun amino-terminal kinase MAPK signalling pathway by SP600125 resulted in no decrease of pollen extractinduced apical CXCL8/IL-8 release. Interestingly, basolateral CXCL8/IL-8 release induced by pollen was not affected by any of the three specific inhibitors of MAPK signalling pathways. Even when applied to the basolateral compartment, the p38 MAPK inhibitor failed to affect basolateral release, even though apical CXCL8/IL-8 release was still inhibited (data not shown). Interestingly, while pollen induced a rapid induction of CXCL8/IL-8 mRNA expression $3 \mathrm{~h}$ after challenge, this was not affected by the p38 MAPK inhibitor (fig. 7b), even though phosphorylation of p38 MAPK was detected as early as 15 min after pollen stimulation in ALI cultures (fig. 7c). These results suggest that p38 MAPK signalling is mainly involved in trafficking of CXCL8/IL-8 to the apical compartment rather than transcriptional control. 

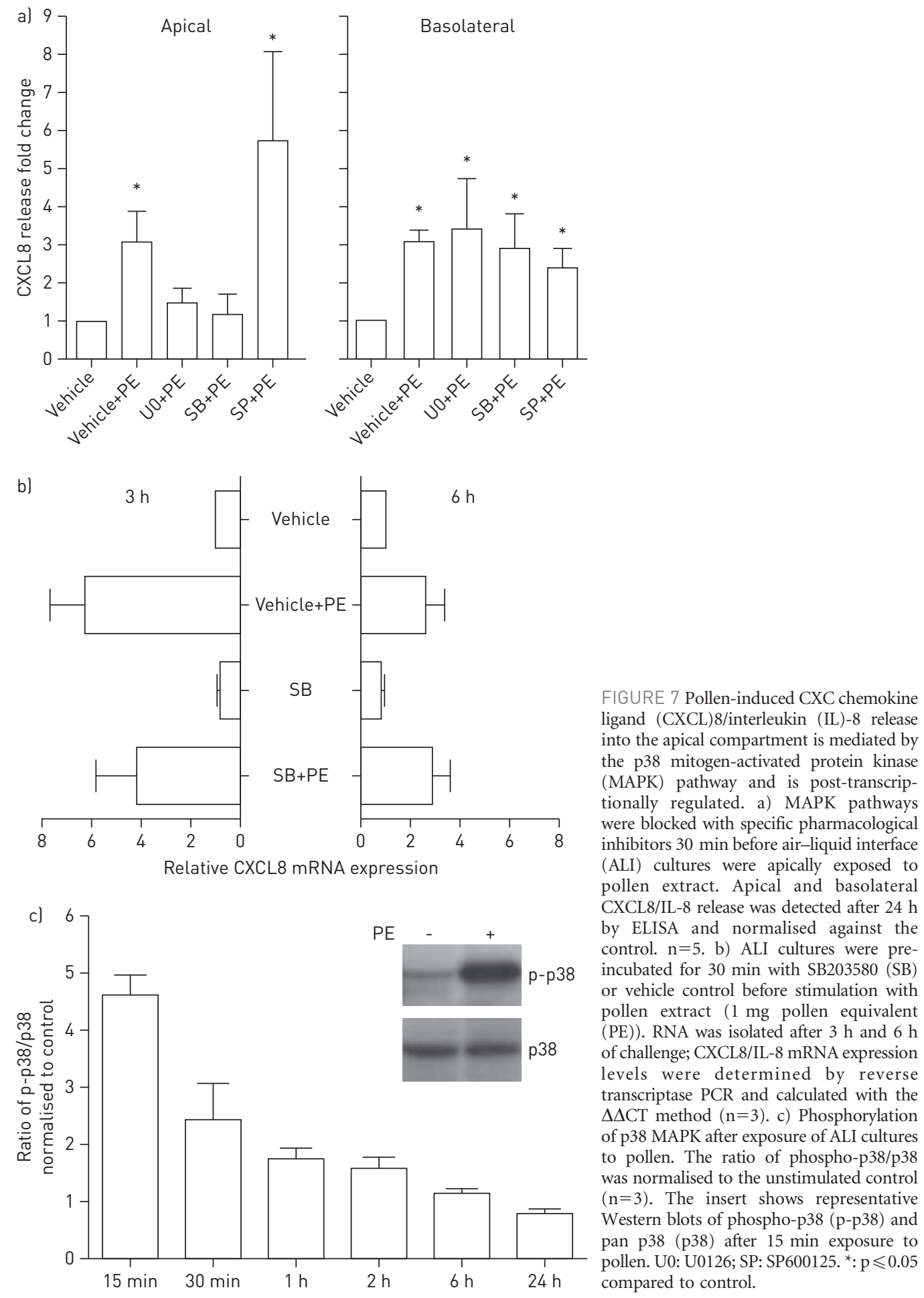

FIGURE 7 Pollen-induced CXC chemokine ligand (CXCL) 8/interleukin (IL)-8 release into the apical compartment is mediated by the p38 mitogen-activated protein kinase (MAPK) pathway and is post-transcriptionally regulated. a) MAPK pathways were blocked with specific pharmacological inhibitors $30 \mathrm{~min}$ before air-liquid interface (ALI) cultures were apically exposed to pollen extract. Apical and basolateral CXCL8/IL-8 release was detected after $24 \mathrm{~h}$ CXISA and normalised against the re preincubated for 30 min with SB203580 (SB) or vehicle control before stimulation with pollen extract ( $1 \mathrm{mg}$ pollen equivalent (PE)). RNA was isolated after $3 \mathrm{~h}$ and $6 \mathrm{~h}$ of challenge; CXCL8/IL-8 mRNA expression were determined by reverse PCR and calculated with the to pollen. The ratio of phospho-p38/p38 was normalised to the unstimulated control $(n=3)$. The insert shows representative (p) rn blots of phospho-p38 (p-p38) and pollen. U0: U0126; SP: SP600125 * : $\mathrm{p} \leqslant 0.05$ compared to control.

\section{Discussion}

Epidemiological studies have demonstrated that the onset of the pollen season, in conjunction with particular climatic conditions (e.g. thunderstorms) is associated with increased risk of asthma exacerbation $[11,12,18]$. Furthermore, even ambient grass pollen levels $<20$ grains $\cdot \mathrm{m}^{-3}$ have been associated recently with increased risk of childhood asthma, independent of any impact of climatic conditions [10]. Grass pollen releases many substances upon hydration, including proteases, allergens and low molecular weight substances with immunomodulatory properties [13]. However, our understanding of effects of particular types of airborne pollen on the airway epithelium, which is the first point of contact with inhaled 
aeroallergens, is limited. Therefore, in the present study, we analysed the impact of grass pollen on the barrier functions of in vitro differentiated PBECs, independently of allergic immune responses. Bronchial epithelial cells differentiated in vitro at the ALI form a structural and functional barrier which includes ciliated and secretory cells [16]. Since the transcriptome of these cultures is comparable to the in vivo situation [19], they represent the best model currently available to analyse barrier functions of bronchial epithelial cells. In our studies, we found that the physical barrier properties of the epithelium were not affected, even after persistent exposure to grass pollen extract over 4 days. However, the cultures did respond to components of pollen extract with vectorial cytokine production. To our knowledge, this is the first report showing that fully differentiated bronchial epithelial cultures are resistant to the potential barrier disrupting effects of the components of pollen extract, even though they release mediators that have the potential to recruit immune cells.

It has previously been reported that proteases and lipid components of pollen extracts have the potential to interfere with the barrier functions of bronchial epithelial cells [14, 15, 20]. For example, pollen from Giant Ragweed, White Birch, Kentucky Blue Grass or Easter Lily have been shown to disrupt epithelial tight junctions with loss of occludin, ZO-1 and claudin-1 from the junctions [15]. However, these studies used polarised, but otherwise undifferentiated cell cultures. One important aspect of ALI cultures is that, as a result of differentiation in the presence of retinoic acid, they make mucous secretions which are similar to the in vivo situation [21]; this enables modelling of interactions between pollen-derived substances and secreted components, such as protease inhibitors, which are able to inactivate environmental proteases. Although we found that the grass pollen extract preparations used in this study contained low levels of protease activity, this failed to affect the epithelial tight junctions when the extracts were applied to the apical surface. In contrast, in the study by RUNSwICK et al. [15], not only were experiments performed on undifferentiated cells in serum-free medium, but extracts equivalent to $50-100 \mathrm{mg} \cdot \mathrm{mL}^{-1}$ of pollen were required to see a barrier-disrupting effect. Considering that extremely high pollen counts are considered to be $>100$ grains $\cdot \mathrm{m}^{-3}$ per day [10] (equivalent to inhaling $>1000$ grains or $>0.5 \mathrm{mg}$ per day), the doses used in the current study (equivalent to $1 \mathrm{mg}$ pollen grains) are a closer approximation to a severe exposure than the previous study. Thus, based on the results of the current study, it is doubtful that grass pollen allergens can disrupt the epithelial barrier in vivo, unless very localised deposition occurs. Consequently, it seems unlikely that pollen-derived allergens can penetrate the tissue via the paracellular route to stimulate immune responses. Although pollen-derived substances can cross the barrier by other mechanisms such as transcytosis [22], the protective mucus layer in the airways may impede both paracellular and transcellular transport. However, unequivocal demonstration that either form of transport does or does not occur would require further experiments using labelled pollen-derived allergens.

Several reports have demonstrated that ALI cultures from severe asthmatic donors retain phenotypic characteristics similar to those observed in vivo. For example, ALI cultures from severe asthmatic donors express more MUC5AC (fig. 1a and [17]) and have impaired barrier properties (fig. 1b and [23]), both as occurs in vivo. Therefore, we also investigated the impact of pollen-derived substances on the barrier functions of differentiated PBECs in severe asthma compared to nonasthmatic controls. However, we found no significant effect on either TER measurements or immunofluorescence staining of ALI cultures from either group after exposure to pollen extract. It is possible that the increase in mucus production observed in the asthma-derived cultures protected the weaker physical barrier of these cultures, although the mucus was washed away before the start of the experiment. Based on the results obtained in this study, we conclude that the impact of grass pollen on the physical barrier function alone is insufficient to account for its effect in asthma.

In the past decade it has become evident that the airway epithelium is an important player in the regulation of immune responses through release of cytokines, chemokines and growth factors [24]. Many reports have shown that the release of chemokines is altered in asthmatic patients at baseline level as well as after bronchial challenge [25-27]. However, most data are based on the analysis of bronchoalveolar lavage fluid or biopsies and therefore reflect the integrated response of many different cell types. Use of in vitro differentiated PBECs provided the opportunity to analyse mediator release from PBECs without interference from other cell types. For baseline CXCL8/IL-8 release, our data are in accordance with previous reports using ALI cultures from paediatric and adult subjects $[7,28]$. In contrast, a recent report has shown an increase in apical CXCL8/IL-8 release in severe asthmatic ALI cultures, which might be a result of slightly different patient populations or culture conditions [17]. However, we observed a significantly higher level of CCL20/MIP-3 $\alpha$ and slightly higher levels of CCL2/MCP-1 and TNF- $\alpha$ in the basolateral mediator release of ALI cultures from severe asthmatic subjects, which have not been reported previously. Increased levels of CCL20/MIP-3 $\alpha$ and CCL2/MCP-1 production in vivo could result in an increase in the migration of immune cells into the airways, where they can amplify the inflammatory immune responses upon activation. TNF- $\alpha$, an important pro-inflammatory cytokine in asthma [29], has also been linked to airway remodelling processes $[30,31]$. Together, even small changes in the levels of only 
a few mediators might change the local milieu in favour of asthmatic inflammation and remodelling. These observations may be further refined by in-depth assessment of the severe asthma phenotypes, which may allow identification of specific cytokines with specific endotypes of the disease.

Due to their ability to form tight junctions, ALI cultures are able to direct vectorial cytokine secretion. Such a polarised release will affect the concentration gradient across the epithelium, offering the potential to promote migration of immune cells across the epithelium into the airway lumen. In the current study, we observed a statistically significant increase in the apical release of CCL20/MIP-3 $\alpha$, CCL22/MDC and TNF- $\alpha$ and a tendency to increased apical release of CCL5/RANTES and CCL2/MCP-1 only after exposure of cultures from severe asthmatic subjects to pollen. It has recently been shown that exposure of an undifferentiated and unpolarised bronchial epithelial cell line to Timothy grass pollen extract increased gene expression or release of CXCL8/IL-8, GM-CSF, CCL2/MCP-1 and CCL20/MIP-3 $\alpha$ [32]. Compared with the relative refractoriness of differentiated cultures from nonasthmatic donors, the similarity of the cytokine profile of the asthma-derived ALI cultures with the undifferentiated model suggests that the asthma cultures may be closer to a wound repair phenotype. In addition to signalling to immune cells, the release of cytokines may also act in an autocrine manner to protect the epithelium. For example, it has recently been shown that the apical release of CCL2/MCP-1 is increased in differentiated PBECs after cigarette smoke exposure and apically administered CCL2/MCP-1 also increases mucus production via apically expressed CC chemokine receptors (CCR)2 [33]. In addition to affecting apical cytokine release, pollen exposure stimulated GM-CSF release, which was directed to the basolateral compartment only in cultures from severe asthmatic patients. This disease-related effect on GM-CSF release is similar to the findings of НACKETT et al. [7], who exposed ALI cultures to respiratory syncytial virus (RSV) or particulate matter. In contrast with the current studies where CXCL8/IL- 8 was released both apically and basolaterally with little effect on CCL5/RANTES, RSV has been shown to trigger release of CXCL8/IL-8 and CCL5/RANTES mainly to the basal compartment [34]. These differences most probably reflect the nature of the stimuli and illustrate the potential for the epithelium to orchestrate (in)appropriate immune responses in health and disease.

Since CXCL8/IL-8 showed the largest increase in the release after pollen exposure of ALI cultures, we analysed the underlying mechanisms that triggered these responses in more detail. We found that ERK1/2 and p38 MAPK signalling pathways were involved in the apical, but not in basolateral, release of CXCL8/ IL-8. Involvement of ERK1/2 and p38 MAPK pathways in CXCL8/IL-8 release has been previously shown by others using different challenges $[35,36]$, but in all these studies cell-line models or undifferentiated PBECs were used. In our analysis, we found that p38 activation and CXCL8/IL-8 mRNA expression occurred rapidly after pollen exposure; however inhibition of p38 MAPK did not affect CXCL8/IL-8 mRNA levels. Failure to inhibit transcription would explain why p38 MAPK inhibition did not affect basolateral CXCL8/IL-8 release, and is consistent with a role for this signalling pathway in CXCL8/IL-8 trafficking to the apical compartment. To date, the underlying mechanisms regulating the transport of proteins to the apical epithelial surface are not fully understood, and apical trafficking of proteins seems to be more heterogeneous than basolateral trafficking [37]. As involvement of MAPK signalling pathways in apical protein trafficking has not been described previously, our findings open the way for further detailed investigations to be performed. A detailed analysis of the mechanisms and effects of a polarised mediator release after exposure of airway epithelial cell to environmental substances will contribute to our understanding of the pathobiology of asthma and assist with development of new and effective therapies.

\section{Acknowledgements}

The authors would like to thank Camelia Molnar (University of Southampton, Southampton, UK) and Gaby PleylWisgickl (Center for Allergy and Environment (ZAUM), Munich, Germany) for their excellent technical assistance.

\section{References}

Holgate ST, Roberts G, Arshad HS, et al. The role of the airway epithelium and its interaction with environmental factors in asthma pathogenesis. Proc Am Thorac Soc 2009; 6: 655-659.

2 Swindle EJ, Collins JE, Davies DE. Breakdown in epithelial barrier function in patients with asthma: identification of novel therapeutic approaches. J Allergy Clin Immunol 2009; 124: 23-34.

Steed E, Balda MS, Matter K. Dynamics and functions of tight junctions. Trends Cell Biol 2010; 20: 142-149. Moffatt MF, Gut IG, Demenais F, et al. A large-scale, consortium-based genomewide association study of asthma. N Engl J Med 2010; 363: 1211-1221.

5 Jacquet A. Interactions of airway epithelium with protease allergens in the allergic response. Clin Exp Allergy 2011; 41: 305-311.

6 Wark PA, Johnston SL, Bucchieri F, et al. Asthmatic bronchial epithelial cells have a deficient innate immune response to infection with rhinovirus. J Exp Med 2005; 201: 937-947.

7 Hackett TL, Singhera GK, Shaheen F, et al. Intrinsic phenotypic differences of asthmatic epithelium and its inflammatory responses to respiratory syncytial virus and air pollution. Am J Respir Cell Mol Biol 2011; 45: 1090-1100.

8 Xiao C, Puddicombe SM, Field S, et al. Defective epithelial barrier function in asthma. J Allergy Clin Immunol 2011; 128: 549-556. 
Forbes L, Harvey S, Newson R, et al. Risk factors for accident and emergency (A\&E) attendance for asthma in inner city children. Thorax 2007; 62: 855-860.

10 Erbas B, Akram M, Dharmage SC, et al. The role of seasonal grass pollen on childhood asthma emergency department presentations. Clin Exp Allergy 2012; 42: 799-805.

11 Dales RE, Cakmak S, Judek S, et al. Influence of outdoor aeroallergens on hospitalization for asthma in Canada. J Allergy Clin Immunol 2004; 113: 303-306.

12 Hayden TJ, Muscatello DJ. Increased presentations to emergency departments for asthma associated with rye grass pollen season in inland NSW. NSW Public Health Bull 2011; 22: 154-158.

13 Gilles S, Mariani V, Bryce M, et al. Pollen allergens do not come alone: pollen associated lipid mediators (PALMS) shift the human immune systems towards a T(H)2-dominated response. Allergy Asthma Clin Immunol 2009; 5: 3.

14 Vinhas R, Cortes L, Cardoso I, et al. Pollen proteases compromise the airway epithelial barrier through degradation of transmembrane adhesion proteins and lung bioactive peptides. Allergy 2011; 66: 1088-1098.

15 Runswick S, Mitchell T, Davies P, et al. Pollen proteolytic enzymes degrade tight junctions. Respirology 2007; 12: 834-842.

16 Gray TE, Guzman K, Davis CW, et al. Mucociliary differentiation of serially passaged normal human tracheobronchial epithelial cells. Am J Respir Cell Mol Biol 1996; 14: 104-112.

17 Gras D, Bourdin A, Vachier I, et al. An ex vivo model of severe asthma using reconstituted human bronchial epithelium. J Allergy Clin Immunol 2012; 129: 1259-1266.

18 D’Amato G, Liccardi G, Frenguelli G. Thunderstorm-asthma and pollen allergy. Allergy 2007; 62: 11-16.

19 Pezzulo AA, Starner TD, Scheetz TE, et al. The air-liquid interface and use of primary cell cultures are important to recapitulate the transcriptional profile of in vivo airway epithelia. Am J Physiol Lung Cell Mol Physiol 2011; 300: L25-L31.

20 Chen-Quay SC, Eiting KT, Li AW, et al. Identification of tight junction modulating lipids. J Pharm Sci 2009; 98: 606-619.

21 Kesimer M, Kirkham S, Pickles RJ, et al. Tracheobronchial air-liquid interface cell culture: a model for innate mucosal defense of the upper airways? Am J Physiol Lung Cell Mol Physiol 2009; 296: L92-L100.

22 Blume C, Foerster S, Gilles S, et al. Human epithelial cells of the respiratory tract and the skin differentially internalize grass pollen allergens. J Invest Dermatol 2009; 129: 1935-1944.

23 Holgate ST. The sentinel role of the airway epithelium in asthma pathogenesis. Immunol Rev 2011; 242: $205-219$.

24 Wang Y, Bai C, Li K, et al. Role of airway epithelial cells in development of asthma and allergic rhinitis. Respir Med 2008; 102: 949-955.

25 Lezcano-Meza D, Negrete-Garcia MC, Dante-Escobedo M, et al. The monocyte-derived chemokine is released in the bronchoalveolar lavage fluid of steady-state asthmatics. Allergy 2003; 58: 1125-1130.

26 Ying S, O'Connor B, Ratoff J, et al. Thymic stromal lymphopoietin expression is increased in asthmatic airways and correlates with expression of Th2-attracting chemokines and disease severity. J Immunol 2005; 174: 8183-8190.

27 Pilette C, Francis JN, Till SJ, et al. CCR4 ligands are up-regulated in the airways of atopic asthmatics after segmental allergen challenge. Eur Respir J 2004; 23: 876-884.

28 Parker J, Sarlang S, Thavagnanam S, et al. A 3-D well-differentiated model of pediatric bronchial epithelium demonstrates unstimulated morphological differences between asthmatic and nonasthmatic cells. Pediatr Res 2010; 67: 17-22.

29 Berry M, Brightling C, Pavord I, et al. TNF- $\alpha$ in asthma. Curr Opin Pharmacol 2007; 7: $279-282$.

30 Kamitani S, Yamauchi Y, Kawasaki S, et al. Simultaneous stimulation with TGF- $\beta 1$ and TNF- $\alpha$ induces epithelial mesenchymal transition in bronchial epithelial cells. Int Arch Allergy Immunol 2011; 155: 119-128.

31 Fujita H, Chalubinski M, Rhyner C, et al. Claudin-1 expression in airway smooth muscle exacerbates airway remodeling in asthmatic subjects. J Allergy Clin Immunol 2011; 127: 1612-1621.

32 Röschmann KI, Luiten S, Jonker MJ, et al. Timothy grass pollen extract-induced gene expression and signalling pathways in airway epithelial cells. Clin Exp Allergy 2011; 41: 830-841.

33 Monzon ME, Forteza RM, Casalino-Matsuda SM. MCP-1/CCR2B-dependent loop upregulates MUC5AC and MUC5B in human airway epithelium. Am J Physiol Lung Cell Mol Physiol 2011; 300: L204-L215.

34 Mellow TE, Murphy PC, Carson JL, et al. The effect of respiratory synctial virus on chemokine release by differentiated airway epithelium. Exp Lung Res 2004; 30: 43-57.

35 Kim YM, Reed W, Lenz AG, et al. Ultrafine carbon particles induce interleukin-8 gene transcription and p38 MAPK activation in normal human bronchial epithelial cells. Am J Physiol Lung Cell Mol Physiol 2005; 288 : L432-L441.

36 Chow AW, Liang JF, Wong JS, et al. Polarized secretion of interleukin (IL)-6 and IL-8 by human airway epithelia 16HBE14o- cells in response to cationic polypeptide challenge. PLoS One 2010; 5: e12091.

37 Weisz OA, Rodriguez-Boulan E. Apical trafficking in epithelial cells: signals, clusters and motors. J Cell Sci 2009; 122: 4253-4266. 